Revue Sciences/Lettres

Sciences / Lettres

1 | 2013

Transferts culturels

\title{
De la traductibilité des Savoirs
}

\section{Charles Alunni}

\section{OpenEdition}

Journals

Édition électronique

URL : http://journals.openedition.org/rsl/293

DOI : $10.4000 /$ rsl.293

ISSN : 2271-6246

Éditeur

Éditions Rue d'Ulm

\section{Référence électronique}

Charles Alunni, «De la traductibilité des Savoirs », Revue Sciences/Lettres [En ligne], 1 | 2013, mis en

ligne le 01 mai 2012, consulté le 01 mai 2019. URL : http://journals.openedition.org/rsl/293 ; DOI : $10.4000 /$ rsl.293

Ce document a été généré automatiquement le 1 mai 2019.

(c) Revue Sciences/Lettres 


\title{
De la traductibilité des Savoirs
}

\author{
Charles Alunni
}

\section{L'Europe ou les savoirs en traduction}

1 La richesse de l'Europe en matière de savoirs provient de la consistance de ses traditions intellectuelles, de ses écoles et d'un savoir-faire inscrits dans des institutions et des langues nationales dont il est raisonnable de supposer une communication et une traductibilité mutuelles à venir.

2 Il importe désormais de créer très rapidement les conditions optimales d'une confrontation rigoureuse de ces expériences. L'un des effets de cette confrontation n'est autre que le renforcement de la prise de conscience de la nécessité vitale dans laquelle on se trouve aujourd'hui, non seulement de penser dans une langue disciplinaire déterminée, mais de s'exprimer également dans d'autres, alors même que la circulation dans l'espace des savoirs, des savoir-faire et des traditions intellectuelles est difficile et que cette difficulté ne peut être contournée.

Il devient dès lors essentiel de développer des questionnements réticulaires et mobiles, où les disciplines ne soient plus étudiées isolément, mais en interactivité permanente, grâce à une pratique transdisciplinaire effective. Une technologie institutionnelle qui le permettrait devrait reposer sur le fait que les disciplines en action doivent aujourd'hui se fonder sur un vaste patrimoine européen laissé en jachère par les deux catastrophes européennes $\mathrm{du} \mathrm{xx}^{\mathrm{e}}$ siècle qu'ont pu représenter les deux guerres mondiales. C'est précisément ce patrimoine qu'avait su exploiter, par exemple, l'Institute for Advanced Study de Princeton au temps de sa fondation, et ce dans les conditions des années 1930 ${ }^{1}$. 


\title{
Communication, diffusion, vulgarisation des savoirs et émergence d'une société de la connaissance
}

\author{
« Je distingue deux moyens de cultiver les \\ sciences : l'un d'augmenter la masse des \\ connaissances par des découvertes; l'autre de \\ rapprocher les découvertes et de les ordonnancer \\ entre elles, afin que plus d'hommes soient éclairés, \\ et que chacun participe, selon sa portée, à la \\ lumière de son siècle. » \\ Diderot
}

4 La communication des savoirs, leur diffusion et leur vulgarisation constituent un des enjeux majeurs de l'émergence d'une société de la connaissance. L'organisation de cette communication suppose, d'une part, le développement d'une réflexion interdisciplinaire sur ses enjeux et, d'autre part, elle engage la constitution de filières de formation à la communication scientifique et à la divulgation des savoirs. Il faut rappeler à cet égard que la nécessité de diffuser la connaissance est affirmée juridiquement depuis la loi d'orientation et de programmation de 1982, par laquelle le législateur a élargi les missions du chercheur à la diffusion des savoirs. Cette dimension est donc essentielle, non seulement dans le contexte des NTIC (Nouvelles technologies de l'information et de la communication), qui participent à l'explosion de la diffusion de l'information scientifique et technique, mais également du point de vue de la lisibilité souhaitée de la recherche scientifique, dans un contexte où la spécialisation disciplinaire restreint tant l'espace intradisciplinaire que l'espace interdisciplinaire, où le chercheur tend à devenir plutôt un homme de technique qu'un homme de culture.

\section{Architecture générale d'une ligne de programme. Écritures et traductions des savoirs}

\section{Le transdisciplinaire et le translinguistique comme enjeux de traduction}

On peut affirmer que la question de la traduction généralisée est le point aveugle du travail philosophique moderne. En effet, il n'existe pas plus de langue unique de la philosophie qu'il n'en existe pour les langages formalisés des sciences « dures ».

6 Il faut que le travail philosophique se fasse à partir de (et en) plusieurs langues, à la fois nationales et disciplinaires. On ne saurait reconduire l'aveuglement de l'empire national d'une seule langue, comme ce fut le cas au siècle dernier.

7 La politique de la traduction traduit toujours une certaine politique : que l'on songe, par exemple, aux divers nationalismes qui ont littéralement miné la philosophie et la science depuis la Première Guerre mondiale.

8 Il s'agit d'une question de travail aux frontières, tout à la fois transdisciplinaires et translinguistiques. 


\section{Cela engage la question du patrimoine savant français et européen}

9 L'intention est de renouer avec une caractéristique propre à la tradition philosophique des langues européennes (France, Italie, Allemagne, Grande-Bretagne), en s'inscrivant réellement dans les thématiques scientifiques contemporaines. Susciter l'intérêt de scientifiques éminents pour la philosophie des sciences doit contribuer à renforcer, croyons-nous, les pôles d'excellence pluridisciplinaires nécessairement inscrits dans leurs grandes traditions généalogiques.

10 La France, l'Italie, l'Allemagne, la Grande-Bretagne, la Suisse ou la Belgique ont représenté, depuis la fin du XIX ${ }^{e}$ siècle jusqu'aux années 1960, la pointe avancée de réflexions, de rencontres et d'écrits fondamentaux pour la compréhension de la science contemporaine.

11 L'une des urgences qui s'impose aujourd'hui à tout philosophe des sciences demeure celle d'exhumer ce patrimoine européen appréhendé comme ensemble de ressources disponibles qu'il faut toujours réactiver en vue de penser notre présent.

12 En se référant à des corpus classiques, et en se rapportant à des conceptions émergentes, on se dirigera vers cet objectif par un quadruple geste méthodologique :

- confrontation à des théories scientifiques récentes ;

- interrogation philosophique des textes scientifiques fondateurs, anciens et contemporains, en leur axe de pensée innovante ;

- exhumation et réactualisation vivifiante de ce patrimoine européen en épistémologie et en histoire des sciences ;

- recours permanent (central et fondamental) à toutes les ressources conceptuelles et catégoriales qui sont à l'œuvre dans l'élaboration de certains dispositifs philosophiques.

13 À moyen terme, il s'agirait de rendre à nouveau disponibles ces savoir-faire en entreprenant la réédition de ces textes fondamentaux de philosophie des sciences, écrits aussi bien par des philosophes que par des scientifiques.

\section{Problématisation et réactivation en temps réel}

14 La recherche reste avant tout une position d'exploration et d'interrogation ancrée dans des savoirs et des savoir-faire partiellement maitrisés. Ce qui correspond à la notion même de question ouverte ou de problématique dans un contexte spécialisé de recherche à un niveau international. Cette problématisation n'a rien d'une réactivation gratuite du philosophème socratique : «Je sais que je ne sais pas. »

La problématisation est au cœur des pratiques scientifiques: elle vit, s'échange, se partage, se métamorphose, prend des couleurs intersubjectives, force les frontières, se prolonge au-delà d'elle-même, se manifeste de manière différente dans tels ou tels esprits qui peut-être n'ont jamais communiqué, mais souvent, de manière inexplicable mais bien réelle, elle apparaît comme leur point de convergence dès lors qu'ils communiquent.

C'est de nouveau en insufflant une distance réflexive et en remontant des généalogies philosophiques encore actives que l'analyse problématisante portera ses fruits, en dynamisant précisément la recherche scientifique comme telle. Certaines de nos attitudes devant l'inconnu sont inspirées, en partie, par le contexte socioculturel dans lequel nous 
nous trouvons, mais également par des mouvements métaphysiques historiques et anciens absorbés par la tradition et la culture présentes.

Une philosophie de la problématisation, articulée à la production contemporaine des sciences, sera en mesure d'éclairer rationnellement ces processus d'influence souterraine. Si les théories achevées et sanctionnées en portent bien trop souvent une trop faible trace, le travail philosophique doit se donner pour tâche de réévaluer ces problématisations, de les faire circuler à tous les niveaux en les saisissant de manière harmonieuse, systématique et vivante, à travers toutes les diagrammatiques possibles du virtuel $^{2}$.

De ces analyses résulte ainsi une approche interne des pratiques scientifiques rendant enfin justice aux discours informels, aux savoirs tacites, aux savoir-faire techniques et gestuels du théoricien comme de l'expérimentateur, aux figurations d'une connaissance approchée, qui permettent aux mathématiciens de communiquer très rapidement des idées qui demandent un travail patient de lecture et d'écriture, d'inscription et de traduction, etc.

L'objectif ici est de pouvoir articuler théoriquement ces pratiques informelles avec les pratiques formelles, et d'approfondir plus encore l'enracinement des concepts dans la «mobilité du géométral ${ }^{3} »$. L'un des dangers du discours par trop machiavélique que pourrait nous inspirer aujourd'hui l'américanisation des pratiques serait de rendre séduisantes des analyses partiales de la science, la représentant de manière réductrice ou réductionniste comme une activité de lutte économique sans merci. Pour ne pas y succomber, il suffit de résister fermement à ces approches superficielles qui sont souvent le fait de sociologies (ou d'histoires des sciences) n'ayant pas fait l'expérience en acte - et traversé l'ascèse - de la recherche en sciences. En se soumettant à la rigoureuse école des sciences, ce projet vise à réhabiliter la réinsertion de la métaphysique et du concept à tous les niveaux, aussi bien pratiques que théoriques, de la science contemporaine.

\section{Tradition - Transmission - Traduction. L'action d'un foncteur universel ${ }^{4}$}

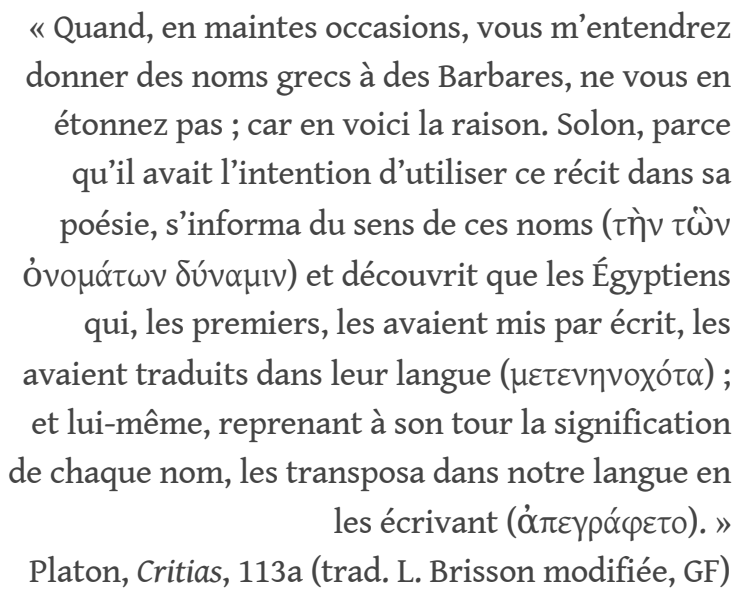

Il semble que la question de la traduction s'impose à partir d'une théorisation de la métaphore, qu'un passage de l'une à l'autre est nécessaire, dicté par la langue même et de l'ordre d'une remontée vers les fondements. 

on constate que la traduction apparaît dans un champ sémantique où s'annonçait la métaphore.

Si ónoүpó $\varphi \omega$ signifie "écrire à partir de », "transposer ", le verbe qui désigne la traduction (très peu courant à l'âge classique) est $\mu \varepsilon \tau \alpha \varphi \varepsilon ́ p \omega$ ("transférer », qui donnera en latin transferre et translatio). La traduction se présente d'abord comme un transfert: Solon traduit en grec ce que les Égyptiens avaient traduit des Barbares, c'est-à-dire leur nom propre. Dans ce cas, la traduction est, par deux fois, perte du propre au profit d'une appropriation.

Avant de déployer mon axiomatique philosophique de la théorie (et de la pratique) de la traduction, je voudrais présenter quelques éclaircissements inauguraux qui impliquent déjà, dès le départ, la traduction à l'œeuvre.

On peut dire que les deux pôles "spectraux" qui condensent au plus près ce dispositif sont d'un côté l'épreuve de l'étranger et, de l'autre, l'idée de "langue en partage". Comment entendre, en notre langue (la "nôtre") et dans la langue de l'Autre (la "vôtre") ces mots habités, hantés par des connotations a priori intraduisibles de manière directe et immédiate, mais constituant leurs ressources sémantiques?

Pour une oreille française, "épreuve" doit s'entendre, tout à la fois :

- comme l'action d'éprouver, qui implique une souffrance (une certaine passivité ?) à l'épreuve de la résistance (de l'Autre, de l'autre langue, du texte de l'Autre) ; épreuve du courage « en traduction ». C'est le lien à l'aventure, au transverse, aux tribulations et au péril'. En son sens guerrier (et toujours empreint d'une certaine passivité), éprouver prend le sens de subir: on dit «éprouver des revers»;

- comme expérience, expérimentation, y compris dans l'ordre de la démonstration: on parlera alors de preuve et de contre-épreuve (d'une théorie par exemple);

- comme ce qui renvoie au sens typographique d'un texte ou d'un manuscrit (ici l'« original») tel qu'il sort de la composition. Corriger et revoir ses épreuves comme on corrige et revoie sa traduction. Ce qui domine en ces deux cas, c'est le risque, ou le soupçon, de la faute (orthographique ou adamique!).

«En partage » s'entend aussi en plusieurs sens, et selon la structure d'un oxymoron:

- cela s'entend d'abord, en français, comme ce qui se donne à partager en commun, ce qui se donne en partage, ne se divise pas (mais pourtant se partage) ;

- le partage s'entend aussi, en notre langue, comme partition, division (ici, au moins en deux, et de deux langues, tenables et intenables à la fois, comme telles, soutenables et insoutenables à la fois - et séparément ;

- le «partage » peut s'entendre également comme supplément (et avec quelque "supplément d'âme» de préférence), au sens de supplément impartageable! Être théoricien de la traduction, traducteur d'(au moins) une langue dans la langue de l'Autre, c'est alors être toujours partagé sur (ou quant à) la langue à partager - avec l'Autre -, sur la «langue en partage ». Par exemple, dans mon cas, entre l'italien et le français, entre la (les) langue(s) philosophique(s), avec leurs techniques terminologiques et syntaxiques propres, et la "langue" scientifique, mathématique ou physico-mathématique.

D'où la question : que signifierait ici «tenir sa langue» dans le partage - maintenir sa langue dans la division et, contemporainement, se taire dans le partage, tenir sa langue pour la partager avec l'Autre? 


\title{
Voies de contrebande
}

\author{
«Écrire quoi que ce soit [...] est un travail de \\ traduction exactement comparable à celui qui \\ opère la transmutation d'un texte d'une langue \\ dans une autre.» \\ Paul Valéry, Variations sur les Bucoliques
}

28 À l'intérieur d'une même langue, le passage de la frontière des genres, le transfert des modèles ou des paradigmes (rhétorique, art, sciences), la situation sur l'« entre-deux » (le mi-lieu) des domaines théoriques et des modèles grammaticaux, constituent des élargissements de la notion de «traduction interne». Ici, comme dans le cas de la traduction "inter-langues », le transfert apparait comme richesse, "plus-value », et non moindre valeur (de sens) ${ }^{6}$.

Reste que la traduction fait toujours problème, car, même lorsqu'on croit savoir ce que "traduction" veut dire, elle échappe à toute maîtrise : telle est l'insistance questionnante de sa pratique et de son « concept ».

Traductio apparait au Moyen Âge dans les écrits théologiques de mouvance aristotélicienne (saint Thomas, Averroès). Ce sens lui sera conservé chez Leibniz, dans sa Théodicée $e^{7}$. Le contexte n'y est pas du tout de l'ordre de l'activité traduisante. Traductio est employé dans le cadre de spéculations sur la forme et la matière, se distinguant dans ce dispositif de l'informatio et de l'eductio. L'informatio, c'est l'imposition d'une forme à une matière (in-former). L'eductio, c'est le dégagement d'une forme existant en puissance dans une matière (la statue dans le marbre). Dès lors, la traductio, c'est le transfert ou la transmission active d'une forme, la forme étant elle-même le principe agissant d'un être. Leibniz donne comme exemple la "traduction des âmes » : " comme si l'âme des enfants était engendrée (per traducem) de l'âme ou des âmes de ceux dont le corps est engendré ». Et l'âme est une forme $e^{8}$. C'est probablement à cette tradition que Leonardo Bruni ${ }^{9}$ prend le terme de traduction, car selon la définition qu'il en donne dans le traité qu'il lui consacre, l'activité traductrice n'est qu'un "transfert » du principe de traduction des âmes au domaine des œuvres (transfert de formes, et non de contenus !). De même, pour Goethe, traduire ne sera rien d'autre qu'engendrer une forme à partir d'une autre forme : méta-morphose.

Le terme de "traduction » appartient, depuis ses débuts romains (et au cœur de sa relève "romantique") à une famille nombreuse, même si elle fut (et reste partiellement) la Cendrillon du groupe. Quelle est cette "famille»? Celle des composés de ducere: conduire, et de ductio : action de conduire. Il n'est pas étonnant que ce peuple si actif devant l'Éternelle traduction qu'étaient les Romains ait créé de nombreux composés à partir de ces deux termes. Le plus marquant est que l'apparition du terme de « traduction » à la fin du $\mathrm{Xv}^{\mathrm{e}}$ siècle soit liée à l'apparition progressive d'autres signifiants en «duction » qui sont tous devenus fondamentaux pour la pensée (et la réalité) en Occident, alors qu'ils n'avaient pas cette importance à Rome : production, reproduction, conduction, déduction, induction, transduction, séduction, introduction, etc. La "traduction " appartient dès lors à l'espace historique de la duction. Tous ces signifiants ont en commun de désigner des processus actifs régis par un sujet. $\mathrm{Si}$ « translation » ne désigne qu'un simple " mouvement » ou "passage ", " traduction » désigne une activité et l'énergie présidant à celle-ci. Comme "production", ce terme dit à la fois le processus et son résultat, ce qui n'est pas vraiment le cas pour « translation $»^{10}$. Désormais, au lieu d'augmenter, comme le 
translator, le traductor innove. Les conséquences pour l'avenir de ce déplacement sémantico-lexical et syntaxique seront incalculables.

Ainsi, le terme de "traduction", comme mauvaise traduction de "traductiontranslation», est bien issu d'une faute de traduction, d'une bévue, d'une "mauvaise lecture »: " traduction " contient en soi, dans son tour et dans son sens, cette «faute " (adamique ?), cette histoire erronée mais productive, car source d'une « nouvelle vie ».

Cette «nouvelle vie », théorisée par Walter Benjamin, est très précisément la "sur-vie » de l'œuvre dans (et par) la traduction : un "plus-de-vie ».

Dans son Aufgabe des Übersetzers, publiée comme préface à sa "propre" traduction allemande des Tableaux parisiens [Heidelberg, 1924], Walter Benjamin dit en substance que la structure même du texte original conçu comme configuration spirituelle, au-dessus et au-delà du simple corps physique du texte, c'est la sur-vie, ce qu'il désigne dans sa langue tantôt comme "über-leben " (survivre comme quelque chose qui s'élève au-dessus de la vie), tantôt comme "fort-leben" (sur-vivre comme quelque chose qui prolonge la vie). C'est, selon des rémanences hégéliennes, la Vie de l'Esprit s'élevant au-dessus de la nature et, comme telle et dans son essence, sur-vie.

La traduction augmente l'original : elle modifie l'original qui, en tant que sur-vie, ne cesse de se transformer, de s'accroître; et elle modifie l'original en modifiant aussi la langue traduisante. Ce processus de transformation de l'original comme de la traduction introduit la structure de contrat de toute traduction ${ }^{11}$. Or, et c'est là l'essentiel pour Benjamin, ce contrat qui n'est ni de représentation, ni de communication, est destiné à assurer, au-delà d'un corpus, d'un texte ou d'un auteur, la survie des langues, la traduction

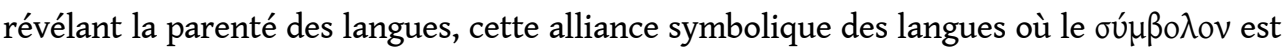
plus grand que l'original lui-même et sa traduction réunis :

Car, de même que les débris d'une amphore, pour qu'on puisse reconstituer le tout ( um sich zusammenzufügen zu lassen), doivent être contigus (einander zu folgen) dans les plus petits détails, mais non identiques les uns aux autres, ainsi, au lieu de se rendre semblable au sens de l'original, la traduction doit bien plutôt, dans un mouvement d'amour et jusque dans le détail, faire passer dans sa propre langue le mode de visée de l'original: ainsi, de même que les débris deviennent reconnaissables comme fragments (Bruchstück) d'une même amphore, original et traduction deviennent connaissables comme fragments d'un langage plus grand $\mathrm{d}^{12}$.

Mais ici les deux moments, celui d'une traduction intra-langue ou interne à une même langue et celui d'une traduction inter-langues, se supplémentent nécessairement. Ce premier sens de la traduction, comme phénomène intralinguistique et "interdisciplinaire ", et le second, plus couramment entendu comme mouvement d'une langue dite naturelle vers une autre, sont rigoureusement liés.

D'où, enfin, une certaine orientation «éthique » (un «habitus» de recherche) de la traduction:

Finalement, j'appelle traducteur, moins un métier, qu'une façon de vivre et de voir le monde. C'est refuser de s'enraciner, c'est rester volontairement dans "l'entredeux" (zwischen). C'est, dans sa façon à lui, un départ. S'il peut y avoir une morale $\mathrm{du}$ traducteur, de la pratique du traduire, eh bien pour moi, ce ne peut être que dans ce refus, dans cet état de suspens qu'il assume de plein gré ${ }^{13}$. 


\section{É(s)trangement ${ }^{14}$ de la langue \& politiques de la traduction}

politique de la traduction, du traducteur et de son effacement (ou encore, de sa clandestinité ): par exemple, dans la détermination des politiques culturelles (modification de la réception par la traduction, déplacement des pôles de savoir) ou éditoriales (pourquoi traduire - ou ne pas traduire - tel corpus, à telle époque, en telle langue ?). Ce qu'on pourrait appeler le «droit d'asile » d'un texte. Je pense ici à la constellation de l'idéalisme allemand, considéré par le philosophe napolitain Bertrando Spaventa comme la simple reprise (la poursuite souterraine et « ailleurs ») d'une textualité nationale et philosophique en exil constituée par la plus pure tradition philosophique italienne (renaissante et moderne), de Giordano Bruno à Gian Battista Vico, en passant par Tommaso Campanella et Galileo Galilei. Pour ce fondateur du néo-hégélianisme napolitain, c'est très précisément cette textualité " originaire " ou "indigène ", traduite une première fois en allemand par celui qui, au cours de ces mêmes années, devient dans son "propre" pays un véritable " chien crevé » (à savoir Hegel), qu'il s'agit de rapatrier dans l'espace "italique » ${ }^{16}$. Ici l'« original» (allemand) n'est original que dans la mesure où il est déjà traduction de la langue traduisante $e^{17}$. Mais si l'idéalisme allemand n'est que la poursuite souterraine et « ailleurs » de la tradition « rinascimentale », c'est qu'il s'agit d'un « retour » de (et par) la traduction, d'une restitution, d'une re-traduction de cette (première?) traduction allemande.

40 Apparaît ici en pleine lumière la structure tératologique de toute "traduction". Pour donner un exemple de traduction "monstrueuse" dont les effets performatifs se révèlent incalculables : Giovanni Gentile, traduisant Feuerbach-Marx et faisant connaître pour la première fois au monde la fameuse $\mathrm{XI}^{\mathrm{e}}$ Thèse, rend « umwälzende Praxis » non par « praxis bouleversante » ou " renversante » (selon un critère matérialiste d'objectivité), mais par " praxis qui se renverse ", constituant ainsi un contre-sens intéressé par subjectivation de la société conçue comme Soi collectif. C'est cette forme auto-référentielle qui autorisera la réduction gentilienne du tout de la philosophie marxiste à la « fureur spéculative » et à sa « métaphysique spéculative ${ }^{18}$.

41 Autre cas, celui de la contrebande linguistique : ainsi lorsque Marx, pendant la rédaction de La Sainte Famille, s'intéresse au matérialisme français, ce n'est point à partir des textes originaux mais à la lumière du Manuel de philosophie moderne de Renouvier (Paris, Paulin, 1842), qu'il traduit-transcrit purement et simplement, déterminant par là tout l'avenir philosophique du matérialisme français ${ }^{19}$.

42 C'est dans le pli d'une telle structure tératologique de la traduction que se pose le problème du transfert des modèles doctrinaux (lesquels? pourquoi ? pourquoi à tels moments dans l'économie générale? pourquoi en un tel lieu?) On peut ainsi parler de trans-duction

Revue Sciences/Lettres, 1 | 2013 
linguistique, de transplantations, de greffes sur une «tradition nationale » déjà là, et des lectures « immanentes » auxquelles ces transferts donnent lieu.

D'où ce problème enfin que connaît tout traducteur : celui du culte et de la rébellion qui se confondent souvent dans sa Stimmung, dans son rapport possible-impossible à l'original, face à cette possibilité toujours impossible de la traduction. Ce qui renvoie à une certaine obsession de l'opacité du langage, paradigmatique chez Kafka, qui énonce ainsi les « impossibilités de langage »:

L'impossibilité de ne pas écrire, l'impossibilité d'écrire en allemand, l'impossibilité

d'écrire différemment. On pourrait presque en ajouter une quatrième:

l'impossibilité d'écrire. ${ }^{20}$ ajoutera dans ses Carnets : « Nous creusons ainsi la fosse de Babel ${ }^{21}$.»

\title{
Transfert mathématique. L'invariant, l'index, la structure, le projecteur et l'archaïque
}

\author{
" "Archaïque" est un terme volontairement \\ provocateur, il renvoie à une existence antérieure, \\ qui précède en quelque sorte le recours à \\ l'axiomatisation.» \\ Alain Connes, Triangle de pensées (éditions Odile
}

Jacob)

Dans l'ouvrage écrit en triangulation avec André Lichnerowicz (physicien-mathématicien et correspondant d'Einstein) et Marcel Paul Schützenberger (spécialiste de combinatoire et de théorie des graphes), Alain Connes a tenté de cerner le concept de réalité (mathématique) archaïque (ou réalité mathématique primitive). Pour cet immense mathématicien, il existe une réalité mathématique qui précède l'élaboration des concepts : c'est ce qu'il définit comme caractère ontologique des mathématiques, caractère que nient les «formalistes" ou "structuralistes » pour qui les mathématiques ne sont qu'un système de déductions logiques obtenues à partir d'axiomes (position incarnée par André Lichnerowicz dans le Triangle). Issue d'une distinction essentielle entre l'objet d'étude (par exemple la "suite des nombres premiers») et les concepts que l'esprit humain élabore pour comprendre cet objet, la réalité mathématique archaïque, c'est l'objet de l'étude : comme la réalité du monde extérieur, elle est a priori non organisée, mais résiste à l'exploration et révèle une cohérence.

La meilleure illustration de cette réalité est l'arithmétique: «Ce que j'appelle "réalité mathématique archaïque" se limite essentiellement aux vérités arithmétiques ${ }^{22}$. " Il est possible de "projeter» tout raisonnement sur l'arithmétique (Gödel). En gros, toute notion mathématique est composée d'une portion qui fait référence à la réalité mathématique archaïque et d'une portion qui est conceptuelle. À cette distinction s'en rattache une autre, celle entre l'«inductif» et le "projectif», qui traverse également toute démarche mathématique. La démarche inductive laisse à chaque objet sa spécificité, son caractère unique (la réalité archaïque); quant à la démarche projective, elle opère sur des objets regroupés par classes (les concepts). «L'idéal se produit lorsqu'on a tellement bien découpé les classes qu'on se trouve devant un objet unique, que l'on connaît de manière à la fois inductive et projective ». Réalité non-matérielle, elle est non périssable 
("aussi résistante qu'un mur»), échappant à toute forme de localisation spatiotemporelle.

47 Le " projectif » est intrinsèquement lié à la notion de structure. Ici «structure » s'entend au sens mathématique, défini ainsi dans son épure extrême par les « formalistes » :

La première opération mathématique consiste - je vais parler le langage des ensembles - à mettre des ensembles en dictionnaires parfaits les uns avec les autres, à ceci près qu'en l'occurrence le dictionnaire est parfait. La notion de structure est alors apparue et leur transfert par ces dictionnaires parfaits a donné la notion d'isomorphisme. [...] Tout ensemble est en un certain sens mathématisable s'il obéit à quelques règles de structure ${ }^{23}$.

Pour Alain Connes, il y a d'un côté cette "réalité archaïque » ou primitive, de nature inductive, extérieure à nous, source inépuisable d'informations, et de l'autre l'outil conceptuel, la division projective en structures que nous élaborons quand nous essayons d'appréhender la réalité archaïque :

Je prétends que nos moyens d'appréhension usuels sont de nature projective. Cette appréhension requiert l'élaboration de concepts de nature projective, puis l'utilisation d'un système logico-déductif, exactement ce que Hilbert a essayé de faire. On met en route la machine de déductions et l'on essaie de déduire ce qui est démontrable à partir des axiomes, etc. [...] C'est un instrument d'observation. Mais je distingue complètement cet instrument d'observation de la réalité qu'il permet d'observer.

Si l'on regarde la suite des nombres premiers [...], elle apparait au premier abord tout aussi bizarre et désordonnée que la réalité extérieure. [...] Grâce à l'élaboration d'un instrument d'observation, grâce à l'invention de concepts appropriés, on arrive graduellement à deviner des régularités structurant cette réalité a priori inorganique [...].

Je prétends qu'en fait les mathématiques se singularisent par rapport à la réalité extérieure par le fait que l'on arrive à caractériser, à singulariser certains objets, par le découpage projectif ${ }^{24}$.

Dès lors, tous les ingrédients d'un dispositif in(-)tra(-)ductif sont réunis et explicitement nommés ${ }^{25}$ :

André Lichnerowicz - Ce que vous appelez « objets », et que j'appellerais volontiers autrement, subsistent dans cette traduction.

Marcel Paul Schützenberger - Comment les appelleriez-vous?

André Lichnerowicz - Je ne sais pas encore. Peut-être des « invariants » ${ }^{26}$.

50 L'enjeu philosophique est ici évidemment majeur. Mais il faut encore clarifier ces concepts relativement familiers au philosophe : celui de "réalité archaïque » bien sûr, mais également ceux, solidaires, d'«invariant» et de "structure». Ce recours aux catégories philosophiques n'est, dans ce contexte d'une pensée mathématique en réflexion, aucunement coupable d'un « coup de force " purement externe :

André Lichnerowicz - Vous êtes en pleine philosophie, vous sortez des mathématiques.

Alain Connes - La philosophie m'intéresse comme moyen de clarifier la pensée, d'éliminer des problèmes qui n'en sont pas pour mettre en évidence les vraies difficultés d'un point de vue ${ }^{27}$.

51 Dans son interprétation de la $\Phi \curlyvee \Sigma I \Sigma$ par le sophiste Antiphon, Martin Heidegger définit ainsi la scène où se mettent en place ces «mêmes » catégories fondamentales mises au jour dans l'ontologie grecque par la «Physique » des Quatre éléments :

Il dit : à la mesure de la púøıৎ est véritablement, et est seulement la Terre, est l'Eau, est l'Air, est le Feu. Mais ainsi, c'est une option décisive qui vient de tomber, une 
ouverture de la plus grande portée : à savoir que ce qui chaque fois apparaît comme davantage, par rapport à la simple Terre (la Terre à l'état pur), par ex. le bois qui se « forme » à partir d'elle, ou bien plus encore le lit fabriqué à partir du bois, tout ce « davantage » est en fait moindrement étant; car ce « davantage » a le caractère de

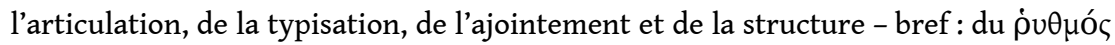

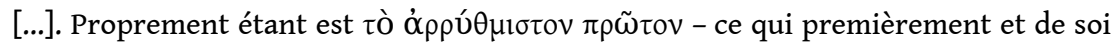
est libre de structure, restant constamment présent au milieu du changement de ce qu'il subit en traversant ses diverses versions et constitutions ${ }^{28}$. haut par André Lichnérowicz, la disposition d'un ordre constitutif (die Verfassung) (ou d'un ordre «projectif » comme le détermine Alain Connes). L'«invariant»- ou la «réalité archaïque »- n'est autre que ce «proprement étant [...] qui premièrement et de soi est libre de structure ». Si l'ordre « projectif » (ce qui relève " de l'articulation, de la typisation, de l'ajointement et de la structure ») est bien ce qui rythme les pulsations du discours mathématicien, s'il en donne la rythmique, alors la «réalité archaïque » se présente

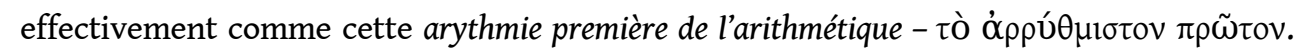
En tant qu' « invariant », elle demeure « constamment présente au milieu du changement de ce qu'elle subit en traversant ses diverses versions et constitutions »!

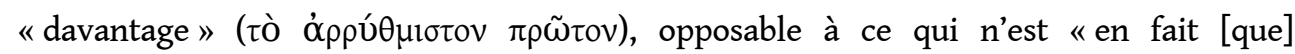
moindrement étant » (die Verfassung) - n'est autre à son tour que ce que Walter Benjamin vise dans ce qu'il appelle, dans le contexte traductif, l'« accroissement des langues » ? Car la « réalité mathématique archaïque » n'opère pas ici autre chose qu'une «traversée » de ses multiples « langues » (ou « structures ») mathématiques, qui tout à la fois la voilent et la dévoilent à travers ce que Connes lui-même donne à lire (à l'instar et à l'insu apparents de Heidegger) comme "ses diverses versions et constitutions »! (a) Sans la structure, pas de visibilité ni de lisibilité "cohérentes », car aucun "instrument d'observation » n'est permis ; (b) avec la structure, apparent coup d'arrêt (et illusion de "clôture ») porté à l'inépuisabilité de cette "source inépuisable ", à la stance (le "restant constamment présent ») «de cette réalité archaïque qui échappera [toujours !] au mode d'exploration donné par la méthode axiomatique et logico-déductive $»^{29}$.

Ici se fondent (sans se confondre) la "sensation de jouissance » du mathématicien ${ }^{30}$ et l'« expérience de la joie » du philosophe face à l'infime « dévoilement »- hors-temps ${ }^{31}$.

Ne semble-t-il pas que de là nous retombions sur la vision «classique » et métaphysique

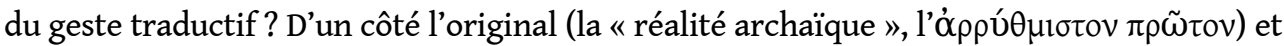
de l'autre la copie (la «structure projective», le $\dot{\rho} \cup \theta \mu o ́ c$, l'articulation traductive «typique » en sa traitrise logico-déductive)?

À une écoute philosophique attentive de la parole mathématicienne, il n'en est rien. C'est ici que convergent précisément les approches déconstructrices de l'héritage métaphysique platonico-aristotélicien - enjeu radical d'une «tra(ns)duction » repensée en termes d'«in(-)tra(-)duction».

Ce qu'il s'agit de lire dans cette traductibilité philosophique des mathématiques, c'est précisément l'affection et l'effraction de la syntaxe accueillante et du dispositif accueilli l'un par l'autre. Le dispositif mathématique - ses algèbres et ses géométries, ses groupes et ses diagrammes - ne laisse pas intact ni vierge l'espace poétique ou philosophique sur lequel il est appliqué. C'est l'enjeu de la transformation - son économie est désormais dominée par l'importation d'une textualité scientifiquement réglée et normativement 
réglementée - ; dans ce cas, plus que jamais, c'est l'enjeu universel de la tra(ns)duction qui dirige les opérations. Or, l'« original » (le texte scientifique) réclame, comme par un contrat invisible (et le plus souvent aveugle), sa (re)traduction philosophique. Et sa traduction ne peut plus être conçue comme une simple transposition (importation passive d'un modèle exogène), il faut la concevoir comme une manifestation "spectrale " des «fantômes " philosophiques qui l'habitent depuis toujours ${ }^{32}$. Le texte "traduisant " constituera dès lors un texte plus grand que la somme de ces fantômes philosophiques et que leur pure et simple juxtaposition ${ }^{33}$. Le "plus-de-vie » sera en ce cas le supplément de mobilités ${ }^{34}$.

Cette position du mathématicien dans cette «triangulation de la pensée " permet déjà de prolonger le tableau résumant l'opération tra(ns)ductive conçue comme opération d'« in (-)tra(-)duction ", c'est-à-dire de brouillage du dispositif métaphysique "classique » de dévalorisation du "geste traductif » et d'effacement de sa puissance performative de production et de création « contractuelles ».

Une connexion philosophique supplémentaire permettrait d'en prendre la mesure pour le champ mathématique, connexion qu'on définira en termes d'affinité sélective avec les approches de Gilbert Simondon ${ }^{35}$, que ce soit autour de la question de l'« individuation » (qu'Alain Connes nomme "singularisation ${ }^{36} »$ ), ou de l'investissement couplé de la "transduction» (qui peut être considérée comme une modalité et une déclinaison de l'«in(-)tra(-)duction", du "passage» d'une réalité mathématique archaïque à ses manifestations « projectives » en structures) ${ }^{37}$.

Reprenant la définition simondonienne, je qualifierai finalement ce prolongement analogique et traductif d'"opératoire " et d'«allagmatique». L' "opération», la " transduction », « l'analogie », le " constructivisme » sont autant de notions subsumées sous ce terme énigmatique. L'allagmatique (du grec $\dot{\alpha} \lambda \lambda \alpha \gamma \eta$ : changement, échange; ó $\lambda \lambda \alpha \gamma \mu \alpha$ (allagma): chose échangée) est avant tout définie comme "théorie des opérations »: elle est complémentaire de la théorie des structures qu'élaborent les sciences. Qu'est-ce qu'une "opération»? "Une opération est une conversion d'une structure en une autre structure ${ }^{38} »$. Aucune opération en-dehors d'une structure ; ainsi, définir une opération consistera pour Simondon à définir une certaine convertibilité de l'opération en structure, et de la structure en opération ${ }^{39}$.

\section{Conclusion par provision}

61 Développer des domaines de recherche nouveaux et les faire circuler auprès de la jeune génération devrait contribuer à dynamiser la philosophie et à favoriser l'émergence de domaines d'étude insoupçonnés. Ce qui devrait contribuer aussi à constituer un large champ d'études comme lieu d'exploration philosophique, permettant de nouvelles visions sur des objets considérés par les mathématiciens (ou les physiciens) professionnels comme classiques, mais que les philosophes ignorent encore largement, à cause d'un manque de formation et de travail. Ainsi, il n'existe toujours pas de travail proprement philosophique et conceptuel mettant en perspective la place progressive de l'algèbre dans l'évolution des idées géométriques, notamment depuis les années 1950, ou la révolution de la Géométrie algébrique ${ }^{40}$. De même, comment dégager les concepts qui gouvernent le développement des théories homologiques et leur diffusion dans l'ensemble des théories physico-mathématiques? 
ces questions sont, depuis 1994, l'objet d'actions transdisciplinaires portées par le Laboratoire disciplinaire «Pensée des sciences » et, depuis 2010, par diverses initiatives conjointes du Centre international de recherches en philosophie, littératures, savoirs $\left(\right.$ Cirphles) ${ }^{41}$.

\section{NOTES}

1. Ed Regis, Who Got Einstein's Office? Eccentricity and Genius at the Institute for Advanced Study [1987], New York, Addison Wesley, 1998.

2. Sur ce point, voir notre article, «Diagrammes \& catégories comme prolégomènes à la question: Qu'est-ce que s'orienter diagrammatiquement dans la pensée?", art. cit.; Gilles Châtelet, L'Enchantement du virtuel. Mathématique, physique, philosophie, éd. Charles Alunni et Catherine Paoletti, Paris, Éditions Rue d'Ulm, 2010.

3. Voir pour cette formule Gilles Châtelet, Les Enjeux du mobile. Mathématique, physique, philosophie, Paris, Le Seuil, 1993, passim.

4. En mathématiques, le foncteur est la généralisation aux catégories de la notion de morphisme, c'est-à-dire d'une application entre deux ensembles munis d'une même structure, et qui respecte cette structure. Le morphisme permet de comparer et de relier les objets mathématiques entre eux.

5. Sur le péril du voyage à l'étranger en son épreuve, voici comment René Descartes en instruisait ses amis : «Je ne sais comment vous pouvez tant aimer l'air d'Italie, avec lequel on respire si souvent la peste, et où toujours la chaleur du jour est insupportable, la fraîcheur du soir mal saine, et où l'obscurité de la nuit couvre des larcins et des meurtres ", À Balzac, 5 mai 1631 ; «Votre voyage d'Italie me donne de l'inquiétude, car c'est un pays fort mal sain pour les Français [...]. Je prie Dieu que vous en puissiez retourner heureusement", À Mersenne, 13 novembre 1639; «J'appréhende pour vous le voyage en Italie que vous vouliez faire cet été [...] », À Mersenne, 29 janvier 1640 ; « Au reste, si vous m'en croyez, vous ne désirerez point faire le voyage d'Italie ; car je ne crois pas que ce soit un pays qui vous soit propre [...] », À Mersenne, 13 octobre 1642.

6. Ainsi, pour prendre un exemple philosophique, Martin Heidegger refusera toujours d'être associé à la "traduction" sartrienne de sa pensée, qualifiant tout rapprochement avec l'existentialisme de contre-sens radical : pourtant, la productivité de ce «malentendu » et de cette "perte », leur performativité comme telles, se sont inscrites dans un plus qui n'est rien de moins que la philosophie existentialiste « elle-même ». On pourrait voir ainsi comment les déformations infligées au «texte-source» performent du nouveau. Sur ce point, voir notre article, "Vues d'Italie ou l'Historicisme en question ", Cahier du Collège International de Philosophie, $\mathrm{n}^{\circ} 2$, Paris, éditions Osiris, 1986, p. 36-40 ; voir également "Attualità, Attuosità », in Barbara Cassin [dir.], Vocabulaire européen des philosophies. Dictionnaire des intraduisibles, Paris, Le Robert/Le Seuil, p. $145-151$.

7. Gottfried Wilhelm Leibniz, Essais de Théodicée sur la bonté de Dieu, la liberté de l'homme et l'origine du mal, Paris, Flammarion, 1999.

8. Ibid., § 86 .

9. Leonardo Bruni (1370-1444) imposa le verbe traducere pour désigner l'acte de «traduction ». Voir son De Interpretatione recta, 1420-1426, traduit pour la première fois en français par nos soins, 
Appendice à Charles Alunni, De la traduction comme pratique théorique, Armand Colin, «Collection U », Paris, 2012.

10. La «translation » est à rattacher au topos médiéval de la translatio studii. La translatio y désigne aussi bien le déplacement physique que le transfert symbolique. Ce sont les connotations de transfert de droit et de transfert métaphorique qui en firent un topos fondateur d'un nouvel ordre culturel. On en doit l'axiomatique à Hugues de Saint-Victor (Didascalicon, III, 2), puis surtout à Chrétien de Troyes (Prologue au Cligès, vers 28 à 42). Dans l'esprit de Saint-Victor, le transfert culturel et la traduction apparaissent indissociablement liés; il va même plus loin en plaçant Cicéron au centre du mouvement de passage du savoir de la Grèce à Rome. Celui-ci non seulement traduit du grec au latin, mais il ajoute de nouvelles composantes au savoir grec. La translatio devient aussi une appropriatio. Chrétien de Troyes déploie l'argument historique du topos : les clercs furent en Grèce, puis émigrèrent à Rome, et maintenant ils sont en France.

C'est le grand Nicolas Oresme qui fera la synthèse de deux siècles d'une élaboration complexe, poussant la logique de la translatio à un degré jamais envisagé par aucun de ses prédécesseurs ou de ses contemporains.

11. Sur la structure de contrat de toute traduction, voir Jacques Derrida, « Des tours de Babel », in Psyché. Invention de l'autre, Paris, Galilée, 1987, p. 220.

12. Walter Benjamin, «La tâche du traducteur », trad. Maurice de Gandillac, Mythe et violence, Paris, Denoël, « Lettres nouvelles », 1971, p. 271-72 ; trad. amendée de M. Broda, Po\&sie, 55, Paris, Éditions Belin, 1991, p. 156.

13. Koitchi Toyosaki (traducteur japonais de Maurice Blanchot, Jacques Derrida, et Philippe Jaccottet), entretien privé.

14. "Estrangement » est un mot du vieux français "estrangier » (encore en usage aujourd'hui au Canada français) qui signifie à la fois étrangement (en son sens actuel) et extranéation (au sens actif). Toujours en usage en langue anglaise estrangement y est défini ainsi : the act of estranging, or the state of being estranged; alienation; separation resulting from hostility (syn: aliénation); the feeling of being alienated from other people (syn : alienation, disaffection); « to estrange » signifie : to make hostile, unsympathetic, or indifferent; to alienate; to remove from an accustomed place or set of associations. Voir sur ce point d'« usage » le titre donné encore récemment à différents ouvrages anglo-saxons: Gerald L. Bruns, Heidegger's Estrangement Language, Truth and Poetry in the Later Writings, New Haven, Yale, 1989 ; Anthony Mellors, « Review of Gary D. Mole, Levinas, Blanchot, Jabes : Figures of estrangement », Oxford Literary Review, 2000, 22, p. 162-177.

15. On en rappelle beaucoup plus souvent les «devoirs»: ici "droit» doit être entendu non seulement eu égard au «traduire » en tant que tel (voir Giovanni Gentile, Du tort et du droit des traductions, Cahier $\mathrm{n}^{\circ} 6$ du Collège international de philosophie, Paris, Osiris, 1988, p. 7-10), mais encore au sens plus proprement juridique du terme. Sur les axiomes comme sur les attendus philosophiques des juristes, voir J. Derrida, «Des tours de Babel » (1980), in Psyché, éd. citée, p. 203-235.

16. Hegel meurt une première fois en 1831 pour devenir très vite le chien crevé de l'Allemagne (seconde mort qui fonde la première). Il trouve alors sa résurrection en Italie, déplacé, transféré, retraduit dans ce qui s'est intitulé Philosophie der Tat, de dérivation fichtéenne et au travers de réformes "subjectivistes" de la dialectique hégélienne (Friedrich Adolf Trendelenburg, Karl Michelet, Karl Werder, Karl Rosenkranz, Johann Eduard Erdmann et Kuno Fischer), en solidarité avec un projet de phénoménologisation politico-programmatique de l'œuvre hégélienne.

17. Voir Bertrando Spaventa, Della nazionalità nella filosofia [Leçon inaugurale prononcée à l'Université de Naples, 23 novembre-23 décembre 1861, réintitulée par son éditeur Giovanni Gentile : La Filosofia italiana nelle sue relazioni con la filosofia europea], Opere, Florence, Sansoni, 1972, vol. II (traduction française par Charles Alunni sous le titre La Philosophie italienne dans ses rapports à la philosophie européenne, Paris, Gallimard, sous presse). Il est à noter que, pour Spaventa, la traduction au sens «strict» renvoie directement (ici comme dans un cas de figure presque 
"pur») au sens extensif du concept de traduction: comme mise en rapport de traditions philosophiques différentes (la «trans-lation» au sens d'Antoine Berman), comme «importexport », puis comme traditionalisation (si ce n'est « nationalisation »).

18. Giovanni Gentile, La Filosofia di Marx (1899), Florence, Sansoni, 1955, p. 85 ; édition française : La Philosophie de Marx, Toulouse, TER, 1995. Sur ce point, cf. André Tosel, Marx en italiques, Toulouse, TER, 1991.

19. À ce propos, voir Olivier Bloch, « Marx, Renouvier, et l'histoire du matérialisme », La Pensée, n - 191, février 1977.

20. Lettre à Max Brod [1921], Franz Kafka, Discours sur la langue yiddish (février 1912). Sur ce conflit babélien, en particulier avec la langue yiddisch, voir Jean-Michel Rey, "L'intraduisible même ", L'Écrit du temps, $\mathrm{n}^{\circ} 7$, ainsi que le très bel article de Régine Robin, «Le Yiddish langue fantasmatique ", L'Écrit du temps, $n^{\circ} 5$.

21. Cité par Jacques Derrida dans L'Oreille de l'Autre. Otobiographies, transferts, traductions, Montréal, VLB, 1982.

22. Alain Connes, Triangle de pensées, Paris, Odile Jacob, «Sciences », 2000, p. 43.

23. Ibid., p. 36.

24. Ibid., p. 46 et p. 54 .

25. Sur notre notion d'«in(-)tra(-)ductif ", qui décline à la fois l'intraductif (comme traduction interne), résultat d'une traduction interne à une même langue ou d'une traduction inter langues, et l'intraductif (comme impossible possibilité de toute traduction), voir notre article « La langue en partage ", Revue de Métaphysique et de Morale, n 1, Paris, Armand Colin, 1989, p. 59-69.

26. A. Connes, Triangle de pensées, éd. citée, p. 57.

27. Ibid., p. 52.

28. Martin Heidegger, Die Physis bei Aristoteles, tr. fr. «Ce qu'est et comment se détermine la Physis? ", Questions II, Paris, Gallimard, p. 525.

29. A. Connes, Triangle de pensées, éd. citée, p. 39.

30. Ibid.

31. Sur ce thème, voir A. Connes, «La réalité mathématique archaïque ", La Recherche, $n^{\circ} 332$, Juin 2000, p. 111 : «Si bien que lorsqu'on en dévoile ne serait-ce qu'une infime partie, on éprouve un sentiment d'éternité. Tous les mathématiciens le savent ».

32. Traduire la science en philosophie fut la tâche bachelardienne par excellence.

33. «Je ne cherche pas à éluder ou éviter les problèmes philosophiques, je considérerais volontiers l'ambition du philosophe comme une ambition de clarté. Je cherche à penser ces problèmes avec des modèles moins réducteurs. Le langage géométrique, qui permet de parler, par exemple, de connexité ou de donner un support précis à une image mentale, devrait donner accès à un contenu plus imaginatif que le langage de la philosophie », A. Connes, Triangle de pensées, éd. citée, p. 198.

34. Sur la thématique de la mobilité (et d'une certaine mobilisation de la pensée), voir G. Châtelet, Les Enjeux du mobile, éd. citée, ainsi que L'Enchantement du virtuel. Mathématique, physique, philosophie, éd. citée, passim.

35. Nous analysons ailleurs ces enjeux fondamentaux, en montrant comment Gilbert Simondon, reprenant de manière singulière l'économie bachelardienne d'une lecture des enjeux philosophiques de la Relativité générale einsteinienne et de la Quantique, réinstaure à nouveaux frais les catégories de «transduction», de «structure ", d'« opération», de "schème », d'« information ", d'« individu», d'« être ", de "pré-individuel " ou d'«ontogenèse », en défondant d'un côté le schème hylémorphique aristotélicien, tout en « réformant » radicalement la dialectique hégélienne en sa triade logique inaugurale (être-néant-devenir), le devenir devenant (dans une impressionnante proximité à la réforme de la dialectique inaugurée par les «petits hégéliens ", puis développée par Spaventa et Gentile) la véritable focale qui rythme la production des «formes", parce que constituant le véritable "centre consistant de l'être ». Sur cette 
économie originale et complexe, voir G. Simondon, L'Individu et sa genèse physico-biologique, Paris, Millon, 1995, Conclusion, p. 228 sq.

36. « Je prétends que les mathématiques se singularisent par rapport à la réalité extérieure par le fait que l'on arrive à caractériser, à singulariser certains objets, par le découpage projectif. [...] Considérons les groupes finis simples par exemple, on en est à un stade de compréhension du monde mathématique tel que l'on dispose de la liste complète de ces groupes. Cela veut dire que les objets qui se trouvent dans cette liste, on peut les nommer, les singulariser, par des propriétés projectives ", A. Connes, Triangle de pensée, éd. citée, p. 54 et p. 53.

37. «Nous entendons par transduction une opération, physique, biologique, mentale, sociale, par laquelle une activité se propage de proche en proche à l'intérieur d'un domaine, en fondant cette propagation sur une structuration du domaine opérée de place en place [...]. La transduction est apparition corrélative de dimensions et de structures dans un être en état de tension préindividuelle, c'est-à-dire dans un être qui est plus qu'unité et plus qu'identité, et qui ne s'est pas encore déphasé par rapport à lui-même en dimensions multiples ", G. Simondon, L'Individu et sa genèse physico-biologique, éd. citée, p. 30-31. On peut conjuguer ici l'«être pré-individuel » simondonien à la " réalité archaïque » de Connes, "source inépuisable d'information ".

38. Sur cette idée de "conversion » comme passage tra(ns)ductif, voir le texte remarquable du bourbakiste André Weil (frère de Simone Weil), De la métaphysique aux mathématiques, in Cuvres scientifiques/Collected Papers, vol. I (1926-1951), New York/Heidelberg/Berlin, Springer Verlag [1960], 1979.

39. L'allagmatique qui saisit l'être antérieurement à toute distinction ou opposition de l'opération et de la structure est la construction d'un point de vue qui comprend l'individu en tant que ce en quoi une opération peut se reconvertir en structure et une structure en opération. Elle s'intéresse aux changements d'état ou à la relation définie comme un aspect de la résonance interne [à entendre aussi au sens précis de la physique nucléaire] d'un système d'individuation.

40. Je citerai, à titre d'exception remarquable, le récent travail de Jean-Pierre Marquis, From a Geometrical Point of View. A Study of the History and Philosophy of Category Theory, Berlin, Springer, 2009. Pour le passé, encore loin d'être épuisé, il faut renvoyer sur ces questions fondamentales aux travaux d'Albert Lautman.

41. http://canoe.ens.fr/ alunni/ \& http://cirphles.ens.fr/

\section{RÉSUMÉS}

Cet article pose le problème de la transmission des savoirs, entre générations, entre communautés savantes linguistiques, et entre disciplines. Cela engage toute une réflexion sur les concepts de transfert et de traduction.

This article raises the problem of the transmission of knowledge between generations, between linguistic and academic communities, and across disciplinary fields, which involves a reflection on the concepts of transfer and of translation. 
INDEX

Mots-clés : traduction, langues et savoirs, transmission des savoirs, transdisciplinaire, mathématiques et philosophie

Keywords : translation, language and knowledge, knowledge transmission, transdisciplinary, mathematics and philosophy 